\title{
Nurses Knowledge Assessment Concerning Prevention of Ventilator-Associated Pneumonia
}

\author{
Hussein Jassim Mohammed ${ }^{1}$, Nuhad Mohammed Kassi ${ }^{2}$, YasharAl-Naamy ${ }^{3}$, Ali F. Abdul Hussein ${ }^{4}$, \\ Raheem Malalla ABADi ${ }^{5}$, R.N Kadhim Hussein Jassim ${ }^{6}$ \\ ${ }^{1}$ A. Prof. Community Health Nursing, College of Nursing, University of Babylon, Iraq, ${ }^{2}$ A. Prof. PhD Child \\ Health, Nursing, College of Nursing, University of Babylon, ${ }^{3}$ BSc nursing gradu, ${ }^{4}$ Ph.D/Instructor,Department of \\ Nursing Al-toosi college University Iraq, ${ }^{5}$ Babylon Health directorate/Iraq, ${ }^{6}$ Babylon Health directorate/Iraq
}

\begin{abstract}
(VAP) is defined as pneumonia that develops in mechanically ventilated patients after 48 hours or more of mechanical ventilator support, VAP increases the mortality and morbidity rates of patients and healthcare costs, This study was a descriptive cross-sectional survey. Sample a total sample of 126 nurses was collected for the current study,setting and Participants a convenience sample of graduate nurses was obtained from eight hospitals throughout Babylon. The study was approved by the ethics committees of the Babylon health department and the participating, Researcher used standard a questionnaire that integrated the assessment of nurse's knowledge for prevention of VAP from the Center of Disease Control and Prevention questionnaire was validated by a panel of four specialists in the disciplines of nursing, The questionnaire was administered to the participants in their hospitals by the principle investigator. Nurses were informed that their completion of the questionnaire would be considered as consent to participate.SPSS version 22 was used for data analysis, The study conclude that the mean age of participants in the study was young and female, the majority of the nurses didn't know that the mechanical ventilator humidifier must be changed weekly to prevent VAP. Researcher encourage all Iraqihospitals ICUs nurses to involve inclinical training to improve nurses acquisition and to evaluate the content of the nursing curricula to identify shortcomings in content. It is imperative that VAP-prevention guidelines be integrated in training and teaching within the nursing schools, and that the content and teaching strategies of infection control courses are updated.
\end{abstract}

Key words: Nurses knowledge, Ventilator-Associated Pneumonia.

\section{Introduction}

The Institute for Healthcare Improvement and the American Thoracic Society have developed evidencebased guidelines to prevent Ventilator Associated Pneumonia (VAP) which is a common nosocomial infection that is associated with increased length of hospitalization, VAP affects $10 \%-25 \%$ of patients who are treated in intensive care units for more than two days, Therate of VAP varies between countries, with higher prevalence and mortality rate reported in developing countries ${ }^{1}$ (VAP) is defined as pneumonia that develops in mechanically ventilated patients after 48 hours or more of mechanical ventilator support, VAP increases the mortality and morbidity ratesof patients and healthcare costs Mechanically ventilated patients are at high risk for developing VAP owing to diverse reasons such as the presence of an endotracheal tube, altered sensorium oral inflammation, impaired mucociliary clearance, inhibition of cough response and micro aspiration of secretions ${ }^{2}$. In 2007, the Institute for Healthcare Improvement (IHI) created the Triple Aim, Which focused on "improving the experience of care providers, improving the health of populations and reducing per capita cost of healthcare." Since that time, healthcare systems across the country have ramped up efforts to reduce the incidence of hospital-acquired conditions (HAC), which account for some readmissions, declining health, and rising costs. To some degree, those efforts are paying off. The Department of Health and Human Services (HHS) reported approximately 87,000 fewer inpatient deaths and a $17 \%$ decline in hospital-acquired 
conditions from 2010 to 2014, resulting in a reduction of almost $\$ 40$ billion in healthcare costs ${ }^{3}$

VAP is account for the highest burden of nosocomial infection as well of health care which is of a serious concern today. It has been reported that the incidence of a nosocomial infections in intensive care unit is about 2 to 5 times higher than in the general in-- patient hospital ${ }^{4}$. A systematic literature review and metaanalysis undertaken the use of antiseptics lowers the risk of VAP, though it does not contribute to the reduction in mortality and duration of ICU stay. ${ }^{2}$ The positive effect of preventive measures may decrease with length of time at risk. Moreover, continuous aspiration of subglottic secretions has a favorable effect on the incidence of early onset VAP, but the effects on late-onset VAP are less definite. ${ }^{5}$. Tracheal suctioning is one of the most common invasive procedures performed by nurses on ICU patients and is crucial to reducing the risk of VAP; Tracheal suctioning is traditionally performed using the open suction system which involves disconnecting the patient from the ventilator and introducing a single-use suction catheterconsists of a multi-use suction catheter included within the ventilation circuit, preventing the need for disconnection and the resultant loss of airway pressure. ${ }^{6}$

Knowledge about VAP prevention varied widely among physicians and nurses. Knowledge gaps affect the implementation and monitoring of VAP prevention practices. Continuing education remains imperative, Knowledge of guidelines for prevention of VAP among healthcare workers (HCWs) has been infrequently assessed and mainly in staff nurses only. The gaps between individual knowledge of VAP prevention and actual daily clinical practice have not been compared. ${ }^{7}$

Prevention of VAP is much more cost-effective than treatment which is an essential objective of health care delivery within ICUs. Care bundle protocols are different and controversial in different hospitals, Preventing infections is the daily work of nurses in every hospital. This applies to the critical unit as well where nurses are the forefront of infection prevention either VAP or other. They create and provide the safe environment, take responsibility for nursing care and play a pivotal role in preventing nosocomial infections. American Organization of Nurse Executives (2015) clinical operations, care management, quality and safety, the patient experience, performance improvement and workforce planning. In addition this role has business and financial expertise. The role of the system chief nurse executive (CNE

\section{Expected Nursing Practice}

Collaborate to identify patients where implementation of noninvasive positive pressure ventilation (NIPPV) may be appropriate to prevent the need for intubation

Assess readiness to extubate daily through combined spontaneous awakening trials (SATs: sedation interruption/minimization) and spontaneous breathing trials (SBTs), unless clinically contraindicated

Maintain and improve physical conditioning through early exercise and mobility

Elevate the head of bed (HOB) to $30^{\circ}$ to $45^{\circ}$ unless clinically contraindicated in patients receiving mechanical ventilation, as well as patients at high risk for aspiration

Minimize pooling of secretions above the endotracheal tube cuff by using an endotracheal tube with subglottic suction capability in patients with anticipated intubation greater than 48 to 72 hours Change ventilator circuits only if visibly soiled; do not change ventilator circuits routinely. ${ }^{9}$

\section{Methodology}

Design: This study was a descriptive crosssectional survey. Sample a total sample of 126 nurses was collected for the current study, the sample size determined by the Rawsoft sample size calculator. Based on an estimated response rate of $50 \%, \alpha=0.05$, power $=0.95$; the estimated population of nurses in Iraq/ Babylon is 200, so the required sample size is estimated to be 120 . The current study used a larger sample to enhance the results' external validity.Inclusion Criteria -Undergraduate nursingschool,setting and Participants a convenience sample of graduate nurses was obtained from eighthospitals throughout Babylon. The study was approved by the ethics committees of the Babylon health department and the participating. 
Researcher used standard a questionnaire that integrated the assessment of nurse's knowledge for prevention of VAP from the Center of Disease Control and Prevention questionnaire was validated by a panel of four specialists in the disciplines of nursing and infection control. The questionnaire comprised two parts. Part one included demographic items such as gender and geographic, Part two comprised 20 multiple-choice questions, each containing four choices: the correct answer and three distracters. The four choices included "I don't know" as an option to prevent guessing. For scoring purposes, the questions were graded as 1 for the correct answer and 0 for other responses. Individual totals for the 20 could thus range from 0 to 20 , a higher score indicating a better knowledge level and a score below 10 meaning that the nurses failed the test.(Aloush \& Qadire, 2017)

\section{Data collection procedure:}

The questionnaire was administered to the participants in their hospitals by the principle investigator. Nurses were informed that their completion of the questionnaire would be considered as consent to participate. Nurses were assured that participation is completely voluntary and that their responses in the questionnaire were confidential. The nurses also were informed that they had the right to withdraw from the study any time. The nurses were subject for any emotional, physical or mental harm as a result of participation in the study. Data were collected in the period April -August 2018.

\section{Statistical Analysis}

SPSS version 22 was used for data analysis. The mean scores and frequencies were calculated. An independent sample t-test was used to measure the difference in mean scores between male and female participants and between nurses with previous education in their training courses about VAP management and mechanical ventilators and those without. An ANOVA test was conducted and the difference in mean scores was compared among nurses according to the geographical location of the hospital.

\section{Results of the Study}

Researcher distributed 140 questionnaires, of which only 126 were completed, a total response rate of $98 \%$. Respondents were of different geographical locations and gender. Participating hospitals were of government affiliation. The mean age of participants in the study was $24.6(\mathrm{SD}=2.9)$ for females and $23.7(\mathrm{SD}=2.7)$ for males. (Table 1)

Table 1. Nurses' characteristics

\begin{tabular}{|c|c|c|c|}
\hline \multicolumn{2}{|c|}{ Items } & \multirow{2}{*}{$\begin{array}{l}\text { Frequency } \\
66 \\
60\end{array}$} & \multirow{2}{*}{$\begin{array}{l}\% \\
52 \\
48\end{array}$} \\
\hline 1 & $\begin{array}{l}\text { Gender } \\
\text { Female: } \\
\text { Male: }\end{array}$ & & \\
\hline 2 & $\begin{array}{l}\text { Hospitals location } \\
\text { Center of Babylon: } \\
\text { Peripheral ofBabylon: }\end{array}$ & $\begin{array}{l}78 \\
48\end{array}$ & $\begin{array}{l}61.9 \\
38.1\end{array}$ \\
\hline 3 & $\begin{array}{l}\text { Received educational program and training on Mechanical ventilator in } \\
\text { their undergraduate courses. } \\
\text { Yes: } \\
\text { No: }\end{array}$ & 64 & $\begin{array}{l}50.8 \\
49.2\end{array}$ \\
\hline 4 & $\begin{array}{l}\text { Received educational program and training on Ventilator Associated } \\
\text { Pneumonia (VAP) management in their undergraduate courses } \\
\text { Yes: } \\
\text { No: }\end{array}$ & 65 & $\begin{array}{l}51.6 \\
48.4\end{array}$ \\
\hline
\end{tabular}


Table 2.Preferred guidelines and frequencies of correct answers Preferred:

\begin{tabular}{|c|c|c|}
\hline & Preferred guidelines and frequencies of correct answers: & $n(\%) / n=126$ \\
\hline 1 & Which method of endotracheal tube insertion is preferred to decrease VAP? & $54(42.8 \%)$ \\
\hline 2 & How often is it preferred the ventilator circuits be changed to decrease the risk VAP? & $61(48.4 \%)$ \\
\hline 3 & Which type of humidifiers is preferred to decrease the risk of VAP? & $75(59.5 \%)$ \\
\hline 5 & How often is it preferred the humidifier be changed to decrease the risk VAP? & $68(53.9 \%)$ \\
\hline 6 & Which type of suctioning systems is preferred to decrease the risk VAP? (open vs close) & $45(35.7 \%)$ \\
\hline 7 & How often is it preferred the suction systems be changed to decrease the risk VAP? & $69(54.7 \%)$ \\
\hline 8 & Which type of endotracheal tube is preferred to decrease the risk of VAP? & $71(56.3 \%)$ \\
\hline 9 & Which type of bed is preferred to decrease VAP the kinetic bed vs the standard bed? & $44(34.9 \%)$ \\
\hline 10 & Which patient position is preferred to decrease the risk VAP? & $65(51.6 \%)$ \\
\hline 11 & Which solution is preferred for oral care to decrease the risk VAP? & $61(48.4 \%)$ \\
\hline 12 & How often oral care is preferred to decrease the risk VAP? & $47(37.3 \%)$ \\
\hline 13 & How often is it preferred to assess patient readiness for extubation? & $75(59.5 \%)$ \\
\hline 14 & How often is it preferred to perform spontaneous breathing trial with sedatives turned off? & $68(53.9 \%)$ \\
\hline 15 & When is it preferred to wash hand to decrease the risk of VAP? & $45(35.7 \%)$ \\
\hline 16 & When is it preferred to wear gloves to decrease the risk of VAP? & $69(54.7 \%)$ \\
\hline 17 & At which level endotracheal tube cuff pressure should be kept to decrease the risk of VAP? & $71(56.3 \%)$ \\
\hline 18 & To decrease the risk of VAP, which of the following practices related to antibiotics use is preferred? & $44(34.9 \%)$ \\
\hline 19 & $\begin{array}{l}\text { What is effect of giving peptic ulcer prophylactic (e.g. H2 blockers, Proton pump inhibitors, or sucral } \\
\text { fate) administration on the risk of VAP? }\end{array}$ & $65(51.6 \%)$ \\
\hline 20 & What is the effect of Deep Venous Thrombosis (DVT) prophylaxis on the risk of VAP? & $61(48.4 \%)$ \\
\hline
\end{tabular}

The mean knowledge score for participants was $6.4(32 \%)(\mathrm{SD}=2.9)$, with a range of $16(80 \%)$ to $0(0 \%) .63$ nurses (14.5\%) answered more than half of the questions correctly. Weakness in the knowledge related to mechanical ventilator management was obvious. The majority of the nurses didn't know that the mechanical ventilator humidifier must be changed weekly to prevent VAP, the endotracheal tube's cuff pressure must be kept at the level of 20$30 \mathrm{H} 2 \mathrm{O}$, and that the ventilator circuit must be changed only when it is visibly soiled or for every new patient's admission. Another topic of concern was oral care and suction. On the other hand, students showed a reasonable level of knowledge regarding hand washing and patients' positioning. (Table 2). 
Table 3. Nurses' scores vs. baseline characteristics

\begin{tabular}{|c|c|c|c|c|}
\hline & & \multicolumn{3}{|c|}{ Nurses Scores out of 20} \\
\hline \multicolumn{2}{|c|}{ Items } & Mean (SD) & $\mathbf{t}$ & $\mathbf{p}$ \\
\hline 1 & $\begin{array}{l}\text { Gender } \\
\text { Female: } \\
\text { Male: }\end{array}$ & $\begin{array}{l}6.42(2.8)^{*} \\
6.44(2.9)\end{array}$ & 0.2 & 0.93 \\
\hline 3 & $\begin{array}{l}\text { Received educational program and training on } \\
\text { Mechanical ventilator in their undergraduate courses. } \\
\text { Yes: } \\
\text { No: }\end{array}$ & $6.9(2.8) 5.9(2.9)$ & 2.1 & 0.00 \\
\hline 4 & $\begin{array}{l}\text { Received educational program and training on } \\
\text { Ventilator Associated Pneumonia (VAP) management } \\
\text { in their undergraduate courses } \\
\text { Yes: } \\
\text { No: }\end{array}$ & $6.4(2.9) 6.6(2.9)$ & 1.9 & 0.014 \\
\hline
\end{tabular}

There was a statistically significant difference in knowledge scores between nurses who had received education in VAP on their undergraduate courses and those who had not: $\mathrm{t}(432)=-3.5, \mathrm{p}=0.00$. However, both groups revealed poor knowledge, The former achieved higher scores $(\mathrm{M}=6.9, \mathrm{SD}=2.8)$ than the latter $(\mathrm{M}$ $=5.9, \mathrm{SD}=2.9$ ). There was no statistically significant difference in the scores of male and female nurse, and no statistically significant difference between nurses who had been taught about mechanical ventilators and those others who had not $(\mathrm{p}>.05)$ (Table 3$)$.

\section{Discussion}

Participants had a poor knowledge of VAPprevention can be compared with other nurses that showed poor overall knowledge about infection control guidelines among student nurses in India, Iran and Spain however, my study was more comprehensive as it focus only on VAP prevention guidelines also it provided a broader set of updated VAP prevention guidelines . Mechanical ventilator management, oral care and the endotracheal tube suction items were the major topics of concern among our nurses, again similar to the findings from another study with student nurses in Spain and with newly graduated nurses in United States. On the other hand, participants showed much better knowledge about hand hygiene and the Semi-Fowler position than about other topics, although their performance was still unsatisfactory.

\section{Conclusions}

The study conclude that the mean age of participants in the study was young and female,the majority of the nurses didn't know that the mechanical ventilator humidifier must be changed weekly to prevent VAP,There was a statistically significant difference in knowledge scores between nurses who had received education in VAP on their undergraduate courses and those who had not. Researcher encourage all Iraqihospitals ICUs nurses to involve inclinical training to improve nurses acquisition and to evaluate the content of the nursing curricula to identify shortcomings in content. It is imperative that VAP-prevention guidelines be integrated in training and teaching within the nursing schools, and that the content and teaching strategies of infection control courses are updated. The findings from 
this study may be used as a reference to evaluate the effectiveness of educational interventions in the future

Financial Disclosure: There is no financial disclosure.

Conflict of Interest: None to declare.

Ethical Clearance: All experimental protocols were approved under the College of Nursing and all experiments were carried out in accordance with approved guidelines.

\section{References}

1. Aloush S, Qadire M. Student Nurses' Knowledge about Prevention of Ventilator-Associated Pneumonia. International Journal of Nursing Education. 2017; 9(2): 138.

2. Chacko R, Rajan A, Lionel P. Oral decontamination techniques and ventilator-associated pneumonia. British Journal of Nursing. 2017; 26(11): 594-599.

3. de Mello L, de Lima D. Prevention of ventilatorassociated pneumonia: a case control study. Online Brazilian Journal of Nursing. 2014; 13(suppl I): 419-421.

4. Dakshinamoorthy S. Compliance of Infection Control VAP Bundle in Critical Care Unit Nurses in Tertiary Care Hospital at Chennai. 2018.

5. Labeau S, Vandijck D, Rello J. Evidence-based guidelines for the prevention of ventilatorassociated pneumonia: results of a knowledge test among European intensive care nurses. Journal of Hospital Infection. 2008; 70(2): 180-185.

6. Osti C, Wosti D, Pandey B, Zhao Q. Ventilatorassociated pneumonia and role of nurses in its prevention. Journal of the Nepal Medical Association. 2017; 56(208): 461-468.

7. Perez-Granda M, Munoz P, Heras C. Prevention of Ventilator-Associated Pneumonia: Can Knowledge and Clinical Practice Be Simply Assessed in a Large Institution? Respiratory Care. 2013; 58(7): 1213-1219.

8. American Organization of Nurse Executives. Nurse Executive Competencies: System CNE. The Voice of Nursing Leadership. 2015.

9. Chulay M, Gallagher J. AACN Practice Alert
Prevention of Ventilator-Associated Pneumonia in Adults. American Association of Critical-Care Nurses. 2017; 37(3): 22-26.

10. Senanayake E, Giri R, Gopal S, Nevill A, Luckraz $\mathrm{H}$. Incidence of endotracheal tube colonization with the use of PneuX endotracheal tubes in patients following cardiac surgery. Journal of Hospital Infection. 2017;95(1):81-86.

11. Larsson J, Itenov TS, Bestle MH. Risk prediction models for mortality in patients with ventilatorassociated pneumonia: A systematic review and meta-analysis. Journal of Critical Care. 2017;37: 112-118.

12. Speck K, Rawat N, Weiner NC, Tujuba HG, Farley D, Berenholtz S. A systematic approach for developing a ventilator-associated pneumonia prevention bundle. American Journal of Infection Control. 2016;44(6):652-656.

13. Schreiber MP, Shorr AF. Challenges and opportunities in the treatment of ventilatorassociated pneumonia. Expert Review of Antiinfective Therapy. 2017;15(1):23-32

14. Mohammad H, Kassim N, Suleiman K. Nutritional status of primary school children from low income households in Jordan. Journal of Natural Sciences Research (JNSR). 2013; 3(2): 61-67.

15. Sajet G, Mohammed HJ. Nutritional Habits of Adolescents and Their Nutritional Health Needs. Indian Journal of Public Health Research \& Development. 2018; 9(10).

16. Jassem A, Al-Doori N, Abd-Redha AA. Mothers' Knowledge Towards Prevention of Early Childhood Home-Injuries at Babylon Province, Iraq. Indian Journal of Public Health Research \& Development. 2019; 10(2): 834-842.

17. Abbas ZK, Kassem N, Shalan A. A Study of Mother's Practices toward Immunization for Children under Six Years in Babylon Province. Indian Journal of Public Health Research \& Development. 2018; 9(8).

18. Abed M, Aldoori N. Assessment of Mothers Education and their Knowledge about HomeAccident among Early Childhood Age Group. kufa Journal for Nursing sciences. 2017; 7(1): 89-96 